\title{
Sense-making : un modèle de construction de la réalité et d'appréhension de l'information par les individus et les groupes
}

Meaning Making: A Model of Reality Enactment and an Approach to

Information for Individuals and Groups

Dominique Maurel

\section{OpenEdition}

Journals

Édition électronique

URL : http://journals.openedition.org/edc/2306

DOl : 10.4000/edc.2306

ISSN : 2101-0366

Éditeur

Université de Lille

Édition imprimée

Date de publication : 1 décembre 2010

Pagination : $31-46$

ISBN : 978-2-917562-04-8

ISSN : $1270-6841$

\section{Référence électronique}

Dominique Maurel, «Sense-making : un modèle de construction de la réalité et d'appréhension de l'information par les individus et les groupes », Études de communication [En ligne], 35 | 2010, mis en ligne le 01 décembre 2012, consulté le 10 décembre 2020. URL : http://journals.openedition.org/edc/ 2306 ; DOI : https://doi.org/10.4000/edc.2306

Ce document a été généré automatiquement le 10 décembre 2020.

(c) Tous droits réservés 


\title{
Sense-making : un modèle de construction de la réalité et d'appréhension de l'information par les individus et les groupes
}

\author{
Meaning Making: A Model of Reality Enactment and an Approach to \\ Information for Individuals and Groups
}

Dominique Maurel

1 Une grande diversité de modèles théoriques existe en sciences de l'information pour étudier la manière dont les individus et les groupes appréhendent l'information. Dans l'approche systèmes (system orientation), issue des sciences physiques positivistes, l'information est considérée comme un objet neutre et objectif à délivrer à un utilisateur, et dont les caractéristiques peuvent être découvertes grâce à l'observation empirique (Choo, 2006). Cela s'est souvent traduit par la conception de systèmes d'information documentaire où le contexte propre à l'usager est évacué, où ses besoins et ses utilisations d'information sont peu pris en compte, et où ses pratiques informationnelles doivent s'adapter aux systèmes. L'approche utilisateurs (user orientation), qui s'inscrit dans le constructivisme cognitif, émerge dans les années 1970 et se confirme dans les années 1980 (Dervin et Nilan, 1986 ; Case, 2007). Une de ses prémisses consiste à être attentif aux besoins des utilisateurs et à concevoir des produits et services d'information mieux adaptés à leurs pratiques informationnelles. De plus, avec l'influence des sciences sociales où le savoir est perçu comme un sens élaboré par l'être humain à partir de ses connaissances, ses expériences et l'environnement qui l'entoure, l'information n'est plus considérée comme un objet extérieur à l'utilisateur mais comme une construction personnalisée (Talja et al., 2005 ; Davenport, 2010).

Le sense-making représente l'un des modèles théoriques les plus utilisés en sciences de l'information pour étudier les comportements informationnels ${ }^{1}$. Le modèle élaboré par Brenda Dervin a été adopté par plusieurs chercheurs. En sciences de la gestion, Karl 
Weick a conçu un modèle de sense-making axé sur la création collective du sens en contexte organisationnel. Ce modèle, très utilisé dans les domaines de la gestion et de la communication organisationnelle, suscite de l'intérêt en sciences de l'information, notamment pour la complémentarité qu'il offre avec celui de Dervin.

Cet article examine les modèles de Dervin et de Weick. Il en expose les fondements épistémologiques, les principaux concepts, le champ d'application et les principales caractéristiques. L'objectif consiste à établir des points d'articulation entre ces deux modèles, tout en examinant leur apport et leur rapprochement possible pour étudier la manière dont les individus et les groupes construisent leur réalité et appréhendent l'information.

\section{Le sense-making individuel - Dervin}

4 Le sense-making de Dervin adopte une position épistémologique constructiviste qui met l'accent sur la manière dont les connaissances sont construites par l'individu. Largement diffusé par Dervin, ce modèle a eu un impact significatif sur l'adoption de la perspective constructiviste dans la recherche en sciences de l'information (Talja et al., 2005 ; Davenport, 2010). Le sense-making se veut aussi en continuité avec des approches cognitivistes, l'information étant avant tout comprise et interprétée du point de vue des individus (Davenport, 2010). Le constructivisme social, pour sa part, inclut le lien entre l'individu et son environnement. Cela peut aussi s'appliquer au modèle de Dervin, où la conception même de la situation initiale implique la prise en compte du contexte. L'approche du sense-making permet ainsi d'établir des patterns représentatifs de la manière dont les individus construisent le sens à des moments précis dans le temps et l'espace et, ainsi, d'améliorer les produits et services offerts aux utilisateurs des bibliothèques et autres services d'information (Dervin, 1983a, 1992).

\section{La métaphore du sense-making}

5 Le modèle théorique du sense-making est présenté chez Dervin comme une métaphore caractérisée par trois concepts fondamentaux: situation initiale, manque ou discontinuité, aide ou résultats. Ces concepts se traduisent par trois questions qui résument le comportement informationnel et indiquent le caractère dynamique et itératif du processus de sense-making : qu'est-ce qui manque dans votre situation et qui fait en sorte que vous vous arrêtiez; quelles questions avez-vous ou quelles ambiguïtés ressentez-vous; quel genre d'aide espérez-vous obtenir pour pouvoir continuer ? L'individu est appelé à expérimenter différents types de discontinuités dans une situation prenant place dans un contexte précis. L'aide prend la forme de recherche puis d'utilisation d'information, cette information devant être intégrée et comprise pour que l'individu puisse poursuivre la situation initiale. Dervin introduit aussi le concept du pont (gap-bridging) qui permet de combler le manque : ce pont informationnel résulte de l'analyse et de l'interprétation d'idées, de valeurs, de connaissances et d'expériences afin de permettre à l'individu de progresser (Dervin, 1992). Le processus est évolutif et peut inclure des boucles de rétroaction, puisque la perception de la discontinuité initiale peut changer suite à l'interprétation qui lui est donnée. Par ailleurs, afin d'opérationnaliser son modèle théorique, Dervin le présente aussi comme une méthodologie. Cela se traduit 
par l'élaboration d'une technique d'entrevue modelée sur les composantes de la métaphore.

Dervin met ensuite l'accent sur les pratiques du verbing afin de tendre vers une compréhension globale de l'activité d'information (Dervin, 1983a,b ; Tidline, 2005). Elle prône l'emploi de verbes plutôt que de noms pour exprimer les différentes actions du gapbridging conduisant à la construction du sens. Cela exprime mieux, selon elle, la manière dont les individus utilisent les idées, pensées, attitudes, croyances, valeurs, sentiments, intuitions, histoires ou souvenirs (Dervin et Frenette, 2003). Par le fait même, cela permet de mieux percevoir que les idées se « font » et donnent lieu à plusieurs versions de la réalité. Il y a ainsi une évolution vers un modèle de construction (ou de déconstruction) du sens dans une perspective communicationnelle (Savolainen, 2006).

\section{Champ d'application et influence du modèle}

7 Le sense-making de Dervin a marqué de manière significative la recherche sur les comportements informationnels, en mettant l'accent sur les utilisateurs plutôt que sur les systèmes. Il représente un outil conceptuel d'application large afin de mieux comprendre les pratiques informationnelles et communicationnelles des individus et des groupes dans différents types de situations et de milieux. La difficulté semble consister à adapter le modèle pour étudier le processus de construction de sens des organisations. Le sensemaking de Dervin a été utilisé notamment dans les recherches sur l'utilisation de l'information dans la vie de tous les jours, représenté par le courant du everyday life information seeking. Le contexte dans lequel s'inscrivent les comportements informationnels est reconnu comme un facteur significatif, ce dont témoigne le courant sur la recherche d'information en contexte (information seeking in context). Choo (1998, 2006) propose pour sa part un modèle général d'utilisation de l'information qui intègre plusieurs modèles et théories faisant autorité dans l'étude des comportements informationnels - notamment Dervin (1992), Belkin (1980 ; Belkin et al., 1982a,b), Kuhlthau (1991, 2004), Ellis (1989), Taylor (1991) et Wilson (1999a,b). Ce modèle général inclut différentes composantes cognitives, affectives et situationnelles permettant d'étudier les comportements informationnels d'individus et de groupes.

Plusieurs tenants de l'approche du sense-making affirment l'intérêt à utiliser celle-ci pour analyser le processus de construction de sens non seulement chez des individus, mais chez des groupes d'individus et des organisations (Fisher, Erdelez et McKechnie, 2005). Dans cette perspective, il est intéressant d'examiner le modèle de construction du sens proposé par Weick pour un contexte organisationnel, et d'observer si des rapprochements sont possibles avec le modèle de Dervin.

\section{Le sense-making organisationnel - Weick}

9 Le sense-making de Weick s'inscrit dans le paradigme constructiviste que certains décrivent aussi comme interprétativiste, puisque de la diversité des interprétations données par différents acteurs organisationnels émerge une perception partagée de la réalité et de l'environnement où ils évoluent (Laramée, 2010). La communication entre acteurs organisationnels est perçue comme un processus d'interactions et de significations partagées. L'organisation représente, pour Weick (1995), une réalité que les acteurs organisationnels perçoivent en fonction de leurs schèmes de pensée propres. 
Leurs décisions et actions sont ensuite fondées sur cette perception - ou construction de leur réalité.

« Comment pouvons-nous savoir ce que nous pensons (ou voulons) avant d'avoir vu (ou compris) ce que nous disons (ou faisons) ? $»^{2}$. Pour Weick, cette phrase contient à la fois la recette de la construction du sens (sense-making) et de l'acte de s'organiser (organizing). Si le sens rend possible l'acte de s'organiser, celui-ci à son tour rend possible le sens : on s'organise à travers et pour le sens, et la création de sens permet aux acteurs organisationnels de partager des modèles mentaux, des valeurs et des croyances, de coordonner des activités et d'agir en interrelation. Ils construisent leur réalité (" énactent " leur environnement) à travers les interprétations qu'ils lui donnent. Puisque la création de sens est rendue nécessaire par l'ambiguïté inhérente à l'environnement, elle pourrait bien être, selon Weick (2001), la problématique organisationnelle centrale.

\section{Le modèle interprétatif du sense-making}

Le modèle interprétatif de Weick vise à comprendre comment les organisations, et les groupes de personnes qui y travaillent, font sens de ce qui se produit autour d'elles, et construisent leur réalité à travers un processus de communication et d'interprétation de messages (ou d'information). Cette construction de la réalité constitue le fondement sur lequel s'appuient ensuite les actions et décisions prises. Weick (1995) identifie sept caractéristiques du sense-making : celui-ci se veut un processus ancré dans la construction de l'identité, rétrospectif puisqu'il n'est possible de bien comprendre une action qu'après l'avoir posée, générateur d'environnements modelés par les acteurs organisationnels, social car il s'inscrit dans les valeurs et interprétations partagées par un groupe d'individus, continu et évolutif, basé sur des signaux et des indices et, enfin, vraisemblable pour les acteurs organisationnels.

En se fondant sur ces caractéristiques, Weick (2001) développe un modèle de sense-making comportant cinq étapes non linéaires :

(1) Le changement écologique, ou discontinuité, est lié à une équivoque issue de l'environnement de l'organisation. Chaque changement perçu est sujet à interprétation par les acteurs organisationnels, ce qui a un impact sur l'action à privilégier (Laramée, 2010).

(2) La mise en actes ou " énaction " implique la construction d'une réalité par les acteurs organisationnels. Dans cette perspective, la réalité et l'environnement ne sont pas acquis une fois pour toutes, mais sont sujets à de constantes redéfinitions (Czarniawska, 2005).

(3) La sélection consiste pour les acteurs à choisir parmi les indices et signaux provenant de l'environnement, et parmi les interprétations qui émergent. La sélection représente une source de justification des actions à privilégier, et l'interprétation retenue a un impact sur la perception de la réalité.

(4) La rétention mémorielle permet aux acteurs de retenir les solutions efficaces qui résultent de la création de sens. Ces solutions contribuent à enrichir les cartes cognitives et à élargir le répertoire des connaissances.

(5) Le souvenir permet de réactiver ce qui a été stocké dans les mémoires et les cartes cognitives. L'apprentissage se produit quand les individus remarquent des facteurs négligés au préalable, et négligent des facteurs remarqués précédemment. Mais paradoxalement, l'enrichissement des cartes cognitives peut aussi limiter la perspective des acteurs organisationnels qui appréhendent les discontinuités ultérieures à la lumière des nouvelles connaissances acquises (Czarniawska, 2005). 
13 La conception du sense-making est ici moins centrée sur la découverte que sur l'invention : construire, filtrer, structurer et ainsi transformer le subjectif en quelque chose de plus tangible (Weick, 2001 ; MacIntosh-Murray, 2005). La création de sens devrait ainsi permettre de faire face à l'environnement.

\section{Champ d'application et influence du modèle}

14 Weick est largement cité dans les écrits en sciences de la gestion, pour analyser par exemple divers types d'organisations et de situations à risque. Son modèle de sensemaking a été moins fréquemment utilisé dans les recherches en sciences de l'information. Mentionnons toutefois Solomon $(1997 a, b, c)$ qui s'y réfère pour étudier le volet social des comportements informationnels d'un groupe de participants engagés dans un processus de travail. Choo $(2002,2006)$ l'utilise dans une étude sur la surveillance de l'environnement par les gestionnaires de grandes entreprises, de même que dans une étude sur les organisations apprenantes. MacIntosh-Murray (2005) se fonde sur Weick pour analyser les comportements informationnels de professionnels de la santé. Weick n'ayant proposé aucune méthodologie spécifique, contrairement à Dervin, les chercheurs qui adoptent son modèle utilisent des méthodes et techniques variées.

Le fait que ce modèle de sense-making organisationnel ne soit pas davantage mis à profit dans les recherches en sciences de l'information est peut-être dû au fait que les chercheurs doivent encore en évaluer la portée dans les études de comportements informationnels qui, souvent, mettent l'accent sur les composantes individuelles. Pourtant, une organisation est un regroupement d'individus qui ont des intérêts communs. En ce sens, le choix de l'un ou l'autre modèle semble davantage relever de la position épistémologique des chercheurs dans leur perception de ce que constitue la construction de la réalité et l'appréhension de l'information dans un contexte " énacté ».

\section{Éléments de synthèse des modèles de sense-making}

16 S’il existe des similitudes entre les processus de construction de sens proposés par Dervin et Weick, certains des concepts propres à ces processus soulèvent un certain nombre d'interrogations. À l'origine, le modèle de Dervin s'intéresse surtout à la perspective cognitive individuelle, alors que les individus expérimentent des discontinuités dans une situation donnée. Les actions entreprises (questions, quête d'information) leur permettent d'acquérir de nouvelles connaissances et de produire de nouvelles significations afin de poursuivre leurs activités. Chez Dervin, le recours à l'information est une condition essentielle à la résolution des manques. Weick, pour sa part, étudie la vie de l'organisation en examinant le phénomène du sense-making chez des groupes d'acteurs organisationnels. Le processus de construction du sens, dans ce cas-ci, est provoqué par une interruption ou discontinuité en relation avec l'environnement de l'organisation, et donc avec les dimensions situationnelles de l'activité en cours. Ici aussi, les groupes agissent, à la différence qu'ils n'entreprennent pas nécessairement une quête d'information dans un système documentaire, même si cela n'est pas exclu. Ils sont toutefois attentifs aux indices et signaux présents dans le discours (les messages), et ce, dans une perspective communicationnelle. Les acteurs organisationnels peuvent aussi avoir besoin de puiser dans les croyances, valeurs et routines partagées, tout comme ils peuvent devoir improviser et créer ainsi de nouvelles façons de faire collectives. Tant 
chez Dervin que chez Weick, toutefois, les acteurs puisent à même des systèmes de mémoire qui se traduisent de différentes manières, qu'il s'agisse de mémoire consignée ou non consignée, tacite ou explicite.

Une hypothèse fondamentale du sense-making porte sur la nature discontinue de la réalité qui est sujette à de multiples interprétations, en raison de changements dans le temps, l'espace et la façon dont les humains construisent des ponts interprétatifs pour comprendre une réalité ; par conséquent, la discontinuité elle-même est de nature subjective (Savolainen, 2006). Son identification relève avant tout de l'interprétation des acteurs organisationnels eux-mêmes, tout comme des chercheurs qui observent le processus lors d'études empiriques (Davenport, 2010). Par ailleurs, certains chercheurs remettent en question les composantes mêmes du modèle de sense-making de Dervin en se demandant s'il doit nécessairement y avoir discontinuité dans une situation initiale (Savolainen, 1993, 2006 ; Davenport, 2010). Le concept de situation est lui-même un terme qui reste à définir, puisqu'il semble compris de différentes manières dans les études empiriques existantes. La situation est le plus souvent liée à un contexte particulier, ce qui limite alors la généralisabilité des résultats issus de ces études. Le caractère fortement contextualisé de la situation dans laquelle s'inscrit un phénomène de construction de sens vient ainsi nuancer la position de Dervin, pour qui les concepts de la métaphore du sense-making font office d'universaux comme éléments explicatifs des comportements informationnels (Dervin, 1983a).

Dervin pose d'autres éléments qui rejoignent les prémisses conceptuelles de Weick. Elle affirme qu'une des découvertes du sense-making est que l'acte de communication et de partage des connaissances fonctionne mieux quand les individus s'ancrent dans leurs histoires et leurs cadres conceptuels (Dervin, 1998 ; Savolainen, 2006). Une des prémisses de Weick est que les acteurs organisationnels possèdent une carte cognitive dont les concepts (et les relations entre ces concepts) leur permettent de mieux interpréter leur organisation. La carte causale, forme particulière de carte cognitive, servirait d'outil du sense-making. En effet, les acteurs organisationnels sont amenés à construire du sens dans des situations nouvelles, alors qu'ils imposent une structure sur ces situations en tentant de les associer à des événements passés : les cartes causales servent d'abord de guides dans la construction de sens puis se transforment en produits de sens, et exercent une influence importante sur la manière dont les acteurs organisationnels perçoivent la réalité. Cette réalité perçue fait partie intégrante de la culture organisationnelle qui résulte des patterns dans les façons de faire de l'organisation et de l'interprétation de ceux-ci. Or une des manières de garder en mémoire tant la culture que les cartes causales repose sur l'utilisation d'" histoires ", employées notamment pour élargir le bassin d'expériences quand il est trop risqué de fonctionner par essai et erreur, et ainsi pour accroître l'efficacité de l'organisation. Dervin (1998) affirme aussi que le cœur des hypothèses du sense-making repose sur l'idée que les connaissances d'aujourd'hui sont rarement adaptées aux utilisations que nous aurons à en faire demain ce qui, dans quelques cas, devient une lacune pour l'avenir ; de plus, les idées, cognitions, sentiments, émotions, questions, souhaits, etc. participent à façonner le sense-making. Cela est similaire au concept de carte cognitive de Weick (2001), que les individus enrichissent et mettent à jour.

Des chercheurs remettent toutefois en question le fait que, chez Weick, les significations soient uniquement dans la tête des individus sous forme de cartes cognitives et non en dehors de ceux-ci, par exemple sous forme d'information consignée (documents) ou non 
consignée (messages, discours) (Laramée, 2010). En effet, les significations s'élaborent aussi grâce aux indices et signaux qui émergent du champ informationnel dans lequel évoluent les acteurs organisationnels, et pas seulement sur la base des connaissances passées. Il est également reproché à Weick de ne pas tenir suffisamment compte du pouvoir exercé par le groupe dominant, dans une organisation par exemple, sur la diffusion des valeurs et significations partagées (Laramée, 2010). Cette vision dominante exerce nécessairement une influence sur la construction du sens des groupes. Les études empiriques doivent faire état de ces biais afin de nuancer le fait que la construction du sens chez des individus ne peut faire abstraction de la construction de sens et de l'interaction de groupes influents (Savolainen, 2006 ; Davenport, 2010).

Dervin conçoit son modèle de sense-making comme étant de nature à la fois théorique et méthodologique. Il reste que sa métaphore du sense-making pourrait être explorée par des techniques d'entrevue autres que celle qu'elle préconise. Le modèle de Weick ne prévoit pas de méthodologie spécifique. Considérant que tout devis méthodologique doit avant tout être fondé sur les questions de recherche propres à l'étude empirique concernée, les chercheurs pourraient expérimenter divers types de méthodes de collecte de données et de techniques d'entrevue, tout en respectant les concepts fondamentaux propres à chaque modèle. Il y aurait donc une certaine souplesse dans la mise en oeuvre du sensemaking.

\section{Conclusion}

21 Le processus de construction du sens est avant tout propre à chaque individu puisqu'il est marqué par des facteurs cognitifs et affectifs inhérents à l'être humain, qu'il s'agisse de sa manière de percevoir sa réalité ou encore d'analyser et d'interpréter des éléments d'information. Mais ce processus est également contextuel puisqu'il prend place dans un environnement social, culturel, économique et politique qui caractérise la situation vécue par un individu, un groupe ou une organisation. La démarche individuelle pour appréhender un phénomène ne peut faire abstraction de l'influence de la collectivité. Le modèle de Dervin semble avoir plus de difficulté à rendre compte de la dimension organisationnelle du processus de création de sens, et de la dynamique qui s'établit entre les caractères individuel et collectif de ce processus et du sens créé. Un individu n'est jamais complètement isolé : il est le produit d'une société, de valeurs et de croyances ; il absorbe les courants dominants de son environnement tout comme les courants de sousculture. La perception qu'il a de sa réalité ne peut par conséquent faire abstraction des interactions avec les autres et avec l'environnement. Le processus de construction du sens devrait ainsi rendre compte de la multiplicité des points de vue qui entrent en conflit ou en négociation afin de déterminer l'influence réciproque qui contribue à " énacter » une nouvelle réalité. Dans cette perspective, il pourrait être intéressant d'enrichir la visée constructiviste du sense-making avec les concepts de l'interactionnisme symbolique qui renvoie à l'évolution d'un phénomène émergeant de l'interaction des acteurs humains et non-humains (les systèmes d'information, par exemple) selon les contingences propres à leur environnement (Strauss, 1993).

Par ailleurs, de manière plus pragmatique, il faut aussi réfléchir aux bénéfices concrets qui résultent des études empiriques fondées sur le sense-making (Davenport, 2010 ; Savolainen, 2006). Dans le domaine des sciences de l'information, ces études ont conduit à l'élaboration de plusieurs modèles de comportements informationnels qui rendent 
compte des particularités propres à des groupes spécifiques d'individus. Il faudrait maintenant pouvoir vérifier si ces modèles ont donné lieu à des améliorations dans les produits et services informationnels offerts aux catégories d'utilisateurs étudiés. Le modèle de Dervin est initialement orienté vers l'amélioration des produits informationnels du monde des bibliothèques, notamment les catalogues, avec les défis posés par la classification, l'indexation et le repérage pour les utilisateurs. Le modèle semble suffisamment souple pour pouvoir s'adapter aussi à d'autres types de systèmes d'information documentaires dans les organisations, notamment ceux relatifs au records management ou à la veille stratégique. En matière de conception de systèmes d'information, le caractère holistique du sense-making représente une force du paradigme utilisateurs. Dans cette optique, il serait intéressant d'effectuer des rapprochements entre le sense-making et la socio-informatique de Kling (2005). Cette approche se préoccupe en effet des aspects sociaux des technologies de l'information et de la communication, dans une perspective globale qui tient compte des éléments humains, culturels, politiques, etc. de l'organisation. Une des principales idées de la socio-technique est que les technologies ne peuvent exister isolément, mais doivent être conçues en fonction du contexte social propre à l'organisation où elles sont implantées. Ce contexte social est lui-même formé de l'interaction de différents éléments, dont font partie les acteurs organisationnels. Des études de sense-making portant sur les comportements informationnels en contexte organisationnel pourraient ainsi contribuer à suggérer des mécanismes pour mieux intégrer les pratiques informationnelles individuelles et collectives, de même que les dimensions formelles et informelles de ces pratiques. L'interaction des acteurs, des systèmes, des pratiques informationnelles et des facteurs cognitifs, affectifs et contextuels des comportements, permettrait d'en arriver à un portrait exhaustif de la construction de la réalité et de l'appréhension de l'information en contexte organisationnel.

\section{BIBLIOGRAPHIE}

Belkin, N. J., (1980), Anomalous states of knowledge as a basis for information retrieval, in Canadian Journal of Information Science, $\mathrm{n}^{\circ}$ 5, pp. 133-143.

Belkin, N. J., Oddy, R. N. et Brooks, H. M., (1982a), Ask for information retrieval : Part 1 Background and theory, in Journal of Documentation, Vol. 38, $\mathrm{n}^{\circ}$ 2, pp. 61-71.

Belkin, N. J., Oddy, R. N. et Brooks, H. M., (1982b), Ask for information retrieval : Part II - Results of a design study, in Journal of Documentation, Vol. 38, $\mathrm{n}^{\circ}$ 3, pp. 145-164.

Case, D. O., (2007), Looking for information : a survey of research in information seeking, needs, and behavior, $2^{\mathrm{e}}$ éd., London, Academic Press, xvi-423 p.

Choo, C. W., (1998), The knowing organization : how organizations use information to construct meaning, create knowledge, and make decisions, New York, NY, Oxford University Press, xviii-298 p.

Choo, C. W., (2002), Information management for the intelligent organization : the art of scanning the environment, $3^{\text {e }}$ éd., Medford, NJ, Information Today, xix-325 p. 
Choo, C. W., (2006), The knowing organization : how organizations use information to construct meaning, create knowledge, and make decisions, $2^{\mathrm{e}}$ éd., New York, NY, Oxford University Press, xiv-354 p.

Czarniawska, B., (2005), Karl Weick : concepts, style and reflection, in The Sociological Review, Vol. 53, $\mathrm{n}^{\circ}$ 1, pp. 267-278.

Davenport, E., (2010), Confessional methods and everyday life information seeking, in Annual Review of Information Science and Technology, Vol. 44, pp. 533-562.

Dervin, B., (1983a), An overview of sense-making research : concepts, methods and results, Paper presented at the Annual Meeting of the International Communication Association, Dallas, Texas, May.

Dervin, B., (1983b), Information as a user construct : the relevance of perceived information needs to synthesis and interpretation (pp. 153-183), in Ward S. A. et Reed L. J. (éds.), Knowledge structure and use : implications for synthesis and interpretation, Philadelphia, PA, Temple University Press, xii-681 p.

Dervin, B., (1992), From the mind's eye of the user : the sense-making qualitative-quantitative methodology (pp. 61-84), in Glazier J. D. et Powell R. R. (éds.), Qualitative research in information management, Englewood, CO, Libraries Unlimited, xiv-238 p.

Dervin, B., (1998), Sense-making theory and practice : an overview of user interests in knowledge seeking and use, in Journal of Knowledge Management, Vol. 2, n 2, pp. 36-46.

Dervin, B. et Nilan, M. S., (1986), Information needs and uses, in Annual Review of Information Science and Technology, $\mathrm{n}^{\circ}$ 21, pp. 3-33.

Dervin, B. et Frenette, M., (2003), Sense-making methodology : communicating communicatively with campaign audiences (pp. 233-249), in Dervin B. et Foreman-Wernet L. (éds.), Sense-making methodology reader : selected writings of Brenda Dervin, Cresskill, NJ, Hampton Press, xv-397 p.

Ellis, D., (1989), A behavioural approach to information retrieval system design, in Journal of Documentation, Vol. $45, \mathrm{n}^{\circ}$ 3, pp. 171-212.

Fisher, K. E., Erdelez, S. et McKechnie, L. E. F. (éds.), (2005), Theories of information behavior, Medford, NJ, Information Today, xxii-431 p.

Kling, R., Rosenbaum, H. et Sawyer, S., (2005), Understanding and communicating social informatics : a framework for studying and teaching the human contexts of information and communication technologies, Medford, NJ, Information Today, xx-216 p.

Kuhlthau, C. C., (1991), Inside the search process : information seeking from the user's perspective, in Journal of the American Society for Information Science, Vol. 42, $\mathrm{n}^{\circ}$ 5, pp. 361-371.

Kuhlthau, C. C., (2004), Seeking meaning : a process approach to library and information services, $2^{\mathrm{e}}$ éd., Westport, CT, Libraries Unlimited, xvii-247 p.

Laramée, A., (2010), La communication dans les organisations : une introduction théorique et pragmatique, $2^{\mathrm{e}}$ éd., Québec, Presses de l'Université du Québec, 343 p.

MacIntosh-Murray, A., (2005), Organizational sense making and information use (pp. 265-269), in Fisher K. E., Erdelez S. et McKechnie L. E. F. (éds.), Theories of information behaviour, Medford, NJ, Information Today, xxii-431 p.

Savolainen, R., (1993), The sense-making theory : reviewing the interests of a user-centered approach to information seeking and use, in Information Processing and Management, Vol. 29, $\mathrm{n}^{\circ}$ 1, pp. 13-28. 
Savolainen, R., (2006), Information use as gap-bridging : the viewpoint of sense-making methodology, in Journal of the American Society for Information Science and Technology, Vol. 57, $\mathrm{n}^{\circ} 8$, pp. 1116-1125.

Solomon, P., (1997a), Discovering information behavior in sense making - I : Time and timing, in Journal of the American Society for Information Science, Vol. 48, $\mathrm{n}^{\circ}$ 12, pp. 1097-1108.

Solomon, P., (1997b), Discovering information behavior in sense making - II :The social, in Journal of the American Society for Information Science, Vol. 48, $\mathrm{n}^{\circ} \quad 12, \mathrm{pp} .1109-1126$.

Solomon, P., (1997c), Discovering information behavior in sense making - III : The person, in Journal of the American Society for Information Science, Vol. 48, $\mathrm{n}^{\circ}$ 12, pp. 1127-1138.

Strauss, A. L., (1993), Continual permutations of action, New York, NY, Aldine de Gruyter, xv-280 p.

Talja, S. et al., (2005), " Isms " in information science : constructivism, collectivism and constructionism, in Journal of Documentation, Vol. 61, $\mathrm{n}^{\circ}$ 1, pp. 79-101.

Taylor, R. S., (1991), Information use environments, in Progress in Communication Science, $\mathrm{n}^{\circ} \quad 10$, pp. 217-254.

Tidline, T. J., (2005), Dervin's sense-making (pp. 113-117), in Fisher K. E., Erdelez S. et McKechnie L. E. F. (éds.), Theories of information behaviour, Medford, NJ, Information Today, xxii-431 p.

Weick, K. E., (1995), Sensemaking in organization, Thousand Oaks, CA, Sage Publications, xii-231 p.

Weick, K. E., (2001), Making sense of the organization, Oxford, Blackwell Publishers, xii-483 p.

Wilson, T. D., (1999a), Exploring models of information behaviour : the " uncertainty " project, in Information Processing and Management, Vol. 35, $\mathrm{n}^{\circ}$ 6, pp. 839-849.

Wilson, T. D., (1999b), Models in information behaviour research, in Journal of Documentation, Vol. 55, $\mathrm{n}^{\circ} 3$, pp. 249-270.

\section{NOTES}

1. Un comportement informationnel comporte une série d'activités (définition des besoins d'information, recherche d'information et utilisation d'information) grâce auxquelles l'information acquiert de l'utilité pour un individu ou un groupe.

2. «How can I know what I think until I see what I say ?» (Weick, 1995, 61 ; Weick, 2001, 95 - notre traduction).

\section{RÉSUMÉS}

Le modèle sense-making offre une perspective constructiviste pour l'étude de la relation des individus et des groupes à l'information. La compréhension qu'ont ces derniers d'une situation, de son contexte et de leur résolution s'appuie notamment sur leurs connaissances, leurs 
expériences et leurs valeurs. Celles-ci exercent aussi une influence sur la manière dont ils reconnaissent ou ignorent l'apport d'information, puis analysent celle-ci et l'intègrent à leurs cartes cognitives ; ce faisant, ils délimitent (« énactent ») leur propre réalité dont les frontières circonscrivent leurs décisions et actions. Cet article examine l'approche de sense-making de Brenda Dervin en sciences de l'information et celle de Karl Weick en sciences de la gestion. Il expose les caractéristiques des modèles proposés, de même que les principales implications de la construction du sens chez les individus et les groupes relativement à la recherche et l'utilisation d'information.

Sense-making offers a constructivist perspective in studying the relation individuals and groups have to information. The understanding they have of a situation, its context and its solving is based on their knowledge, their experiences and their values. Those also impact on how they acknowledge or ignore information, and how they analyze it and integrate it to their cognitive maps. By doing so, they define (" enact ») their own reality which then defines the nature of their decisions and actions. This paper examines the sense-making approach of Brenda Dervin in information studies and of Karl Weick in management studies. It describes the components of their models, as well as the main implications of sense-making for individuals and groups relative to information seeking and use.

\section{INDEX}

Mots-clés : construction de sens, constructivisme, recherche d'information, comportement informationnel, modèle, individu, organisation

Keywords : sense-making, constructivism, information seeking, information behaviour, model, individual, organization

\section{AUTEUR}

\section{DOMINIQUE MAUREL}

École de bibliothéconomie et des sciences de l'information - Université de Montréal

Dominique Maurel est professeure adjointe à l'École de bibliothéconomie et des sciences de l'information (EBSI) de l'Université de Montréal. Ses principaux intérêts de recherche portent sur les comportements informationnels dans les organisations, la stratégie informationnelle et les politiques d'information dans les organisations, de même que sur la gestion de la mémoire organisationnelle. Adresse électronique : dominique.maurel@umontreal.ca. 